\title{
Estudo comparativo entre algoritmos das transformadas discretas de Fourier e Wavelet
}

\author{
Wilson Hissamu Shirado ${ }^{1}$ \\ Márcio de Abreu Moreira ${ }^{1}$ \\ Jandira Guenka Palma ${ }^{1}$ \\ Sylvio Barbon Júnior ${ }^{1}$
}

\begin{abstract}
Resumo: Este trabalho apresenta um estudo comparativo das complexidades dos algoritmos das Transformadas Discretas de Fourier, Wavelet e Transformada Rápida de Fourier. As formalizações matemáticas e algumas características dos algoritmos são apresentadas, assim como alguns conceitos de complexidade assintótica. Por fim, é realizado um ensaio prático para comparação dos algoritmos, abrangendo questões como tempo de execução, vantagens e desvantagens de cada transformada assim como avaliações a respeito das diferentes resoluções tempo/frequência de cada algoritmo.
\end{abstract}

Palavras-chave: Complexidade Computacional. Processamento Digital de Sinais. Transformada de Fourier. Transformada Wavelet. Fast Fourier.

\begin{abstract}
This paper show a comparative study between Discrete Fourier Transform, Discrete Wavelet Transform and Fast Fourier Transform. The math formalizations and some features of algorithms are showed and asymptotic complexity concepts. Finally, is performed a practical experiment for comparing algorithms, covering issues as executing time, pros and cons of each transformed, as well as evaluate about the diferent resolutions time/frequence of each algorithm..
\end{abstract}

Keywords: Computational complexity. Digital signal processing. Fourier Transform. Wavelet Transform.

\section{Instrução}

O Processamento de Sinais - PS, é uma área da tecnologia que analisa ou modifica um sinal a fim de extrair informações ou adequá-lo a uma aplicação específica, podendo ser feita de forma analógica (tempo contínuo) ou digital (tempo discreto). Tais técnicas podem ser aplicadas a diversos tipos de sinais como imagens, áudio, ondas elétricas, fluxo de rede entre outros. Dessa forma, o PS contribui em diversas áreas do conhecimento, tais como comunicação [1], medicina [2], ecologia e meio ambiente [3], meteorologia [4], sendo, portanto, um assunto bastante explorado em pesquisas atuais.

Em termos computacionais um sinal geralmente é discretizado por meio de hardwares específicos, como por exemplo, uma placa de áudio ou vídeo, sendo assim transformado em um sinal digital. Uma vez que o sinal foi digitalizado, torna-se possível a aplicação das técnicas de PS para finalidades diversas, como análises, compressões, filtragens entre outras. As técnicas de PS são realizadas a partir da implementação e da execução de algoritmos que realizam transformações e permitem a análise dos sinais digitais.

Dessa forma, esse trabalho apresenta uma abordagem para compreensão, disseminação de conceitos, teorias e técnicas utilizadas no PS. Dentre as diversas técnicas de PS existentes, destaca-se o uso de ferramentas matemáticas para representação e manipulação de sinais discretos no tempo e espaço, como a Transformada Discreta de Fourier (DFT), a Transformada Rápida de Fourier (FFT) e a Transformada Discreta Wavelet (DWT). Este artigo tem como objetivo realizar um estudo comparativo entre os algoritmos dessas, abordando questões relacionadas à complexidade computacional, vantagens e desvantagens de cada algoritmo, assim como suas

\footnotetext{
${ }^{1}$ Programa de Pós-graduação em Ciência da Computação, Universidade Estadual de Londrina -UEL, Departamento de Computação-DC, Bloco J, Sala 305a , Cx. Postal 10011- CEP: 86057-970 - Londrina (PR) - Brasil

\{hissamu.shirado, marcio.abrm\}@gmail.com, \{jgpalma, barbon\}@uel.br
}

http://dx.doi.org/10.5335/rbca.2015.4880 
aplicações em contextos diversos. Serão analisados os algoritmos da DFT, FFT e quanto a DWT será analisado o algoritmo utilizando a família Wavelet de Haar, a mais simples das famílias.

Este trabalho foi organizado de maneira que, na segunda seção, são apresentados os conceitos e referenciais teóricos necessários para a melhor compreensão do assunto abordado. Na terceira seção, a metodologia adotada para a realização das comparações entre as transformadas discretas, e na seção 4 e 5 , serão discutidos os resultados e conclusões respectivamente.

\section{Referencial Teórico}

O Processamento Digital de Sinais - PDS é um campo com vasta gama de aplicações e pesquisas em variadas áreas de conhecimento. Dessa forma, serão abordados nesta seção trabalhos relacionados às transformadas, os principais conceitos relativos ao PDS, assim como os conceitos pertinentes a algoritmos, complexidade computacional assintótica e as respectivas formulações matemáticas.

\subsection{Trabalhos Relacionados}

Aplicações de PDS são encontradas nos mais variados campos de pesquisa e situações cotidianas. Dois exemplos práticos e comuns são o algoritmo aplicado na compressão de áudio para o formato mp3, que utiliza a Discrete Cosine Transform - DCT [5] e o algoritmo aplicado para compressão de imagens no padrão JPEG2000 que utiliza a DWT [6].

Além disso, as técnicas de PDS são aplicadas à resolução de problemas científicos diversos como a aplicação da FFT para auxiliar a análise de imagens médicas da retina de pacientes com glaucoma com o intuito de detectar defeitos localizados na camada de fibras nervosas da retina [7]. Outro trabalho de pesquisa relacionada à saúde, registrou bons resultados utilizando a DFT para o processamento e a reconstrução de superfícies em imagens de exames de Tomografia de Fluorescência Molecular [8].

Além dos trabalhos voltados à Transformada de Fourier, existem outros estudos, realizados a partir da aplicação das DWT's, como o trabalho apresentado em [9], no qual os autores fazem uso dela em conjunto com Redes Neurais Artificiais para o reconhecimento de expressões faciais em indivíduos. Outros trabalhos recentes propõem a utilização das DWT's para a compressão, o armazenamento e a transmissão de imagens 3D [10].

A análise da complexidade de algoritmos é uma forma de avaliar e mensurar o esforço computacional exigido na execução de um algoritmo. Esta análise auxilia na definição do algoritmo mais adequado para resolução de um problema específico. Exemplo disso é o estudo realizado no setor de telecomunicações que avalia os algoritmos de detecção de espaços espectrais brancos para aplicações de rádio cognitivo [11]. Outro trabalho apresenta uma análise da variação dinâmica da complexidade de um algoritmo de tracking, voltado à área de visão computacional, com objetivo de reduzir tempo de processamento e aprimorar a qualidade do algoritmo [12].

\subsection{Análise de Algoritmos}

O PDS por computadores é possível devido aos algoritmos computacionais que são abstrações de operações matemáticas para resolver um problema em um número finito de passos que frequentemente envolvem a repetição de uma operação [13], que podem propiciar a avaliação da complexidade do algoritmo. A análise de algoritmos baseia-se principalmente no estudo da complexidade desse, sendo importante a avaliação da taxa de crescimento do tempo de execução como uma função matemática baseada no tamanho da entrada $n$. Desta forma se obtêm um quadro geral do comportamento desse algoritmo relevando-se os detalhes menores. Estruturas de dados e algoritmos são normalmente analisados usando-se uma notação matemática para funções que desconsidera os fatores constantes. Desta forma, o tempo de execução de um algoritmo será caracterizado usando funções de crescimento assintótico que mapeiam o tamanho da entrada $\mathrm{n}$, para valores que correspondem a um fator principal que determina a taxa de crescimento em termos de $n$ [14].

\subsection{Notação $O$}

A notação $O$ é usada para caracterizar o tempo de execução e os limites espaciais em função de um parâmetro n que varia de problema para problemas, mas é geralmente definido como uma medida escolhida do 
tamanho desse. Sejam $f(n)$ e $g(n)$ funções mapeando inteiros não-negativos em números reais, entende-se que $f(n)$ é $O(f(n))$ se existe uma constante real $c>0$ e uma constante inteira $n_{0} \geq 1$ tais que $f(n) \leq c g(n)$ para todo inteiro $n \geq n_{0}$. Esta é uma definição da notação $O$, onde $f(n)$ é $\mathrm{O}$ de $g(n)$. Outra forma de entendimento diz que $f(n)$ é da ordem de $g(n)[14]$.

\subsection{Complexidade dos Algoritmos}

Segundo [15], os algoritmos podem ser classificados quanto à sua complexidade computacional como constante, logarítmica, sublinear, linear, $n \log (n)$, quadrática e exponencial. Na Tabela 1, é possível verificar algumas complexidades, suas funções básicas e uma breve descrição.

Tabela 1: Complexidade Algorítmica

\begin{tabular}{lll}
\hline Complexidade & Função & Descrição \\
\hline Constante & $O(1)$ & $\begin{array}{l}\text { Independe do tamanho n de entradas, as instruções são executadas } \\
\text { em um número fixo de vezes. }\end{array}$ \\
Logarítmica & $O(\log n)$ & $\begin{array}{l}\text { Divide um problema em problemas menores. } \\
\text { A quantidade de passos aumenta linearmente em relação ao tamanho } \\
n \text { de entradas }\end{array}$ \\
Linear & $O(n)$ & $\begin{array}{l}\text { Divide o problema em problemas menores, porém junta } \\
\text { posteriormente a solução dos problemas menores. }\end{array}$ \\
n log (n) & $O(n \log n)$ & $\begin{array}{l}\text { Geralmente com um laço dentro do outro. } \\
\text { Itens são processados três a três, geralmente com um laço dentro dos } \\
\text { outros dois. }\end{array}$ \\
Cúbica & $O\left(n^{2}\right)$ & $\begin{array}{l}\text { São soluções simples de um determinado problema. Devido à alta } \\
\text { complexidade, não são úteis sob o ponto de vista prático. }\end{array}$ \\
\hline
\end{tabular}

Fonte: $[14,16]$

A complexidade de um algoritmo pode ser definida pelo número de operações matemáticas realizadas e a quantidade de vezes que se repetem durante a execução do algoritmo.

O tempo de execução e o espaço em memória utilizado são algumas medidas que tentam expressar a eficiência de um algoritmo. Porém avaliar o desempenho de algoritmos pelo tempo é fortemente dependente tanto do programa quanto da máquina usada para executar o algoritmo. Por exemplo, em uma situação na qual um computador A executa um bilhão de instruções por segundo e o computador B executa apenas dez milhões de instruções por segundo, o computador A tende a ser 100 vezes mais rápido que o computador B em capacidade bruta de computação, influenciando assim o tempo de execução do algoritmo [16].

Com relação ao tempo, deve-se considerar a taxa de crescimento da execução em função do tamanho da entrada, como é apresentado na Figura 1. 
Figura 1: Comparativo da complexidade das funções

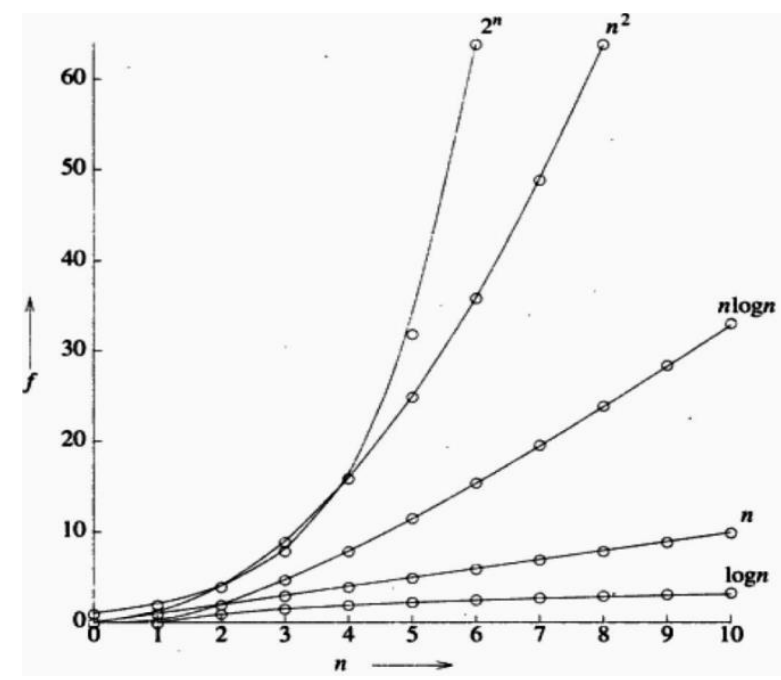

Fonte: [17]

\subsection{Processamento Digital de Sinais e Amostragem}

Antes do surgimento dos computadores e os algoritmos computacionais, o tratamento de sinais era realizado de forma analógica trabalhando com sinais no tempo contínuo. No entanto, com a criação dos microprocessadores e a evolução das abordagens foi possível realizar o processamento dos sinais da forma digital permitindo trabalhar com sinais em tempo discreto.

A discretização de um sinal analógico pode ser realizada por meio da operação de amostragem $x[n]=$ $x_{c}\left(n T_{a}\right)$, onde $x[n]$ é um vetor (sinal) discreto que armazena $n$, a amostra, $x_{c}(t)$ é a função analógica (sinal contínuo) e $T_{a}$ é o período de amostragem [18].

\subsection{De Fourier a Wavelet}

O matemático e físico francês Joseph Fourier, autor da Teoria Analítica do Calor de 1822 desenvolveu diversas pesquisas na área de PS. Ele criou as Séries de Fourier com intuito inicial de estudar a condução do calor. Em sua teoria ele demonstrou que qualquer função periódica pode ser expressa por uma somatória de senos e cossenos [19].

Os estudos de Fourier serviram como base para outros pesquisadores realizarem seus trabalhos e criações, um exemplo disso é o surgimento da DWT, que evoluiu dos conceitos de Fourier em conjunto com outras técnicas o que possibilitou análises em diferentes escalas de frequência e de tempo, diferente de Fourier que proporciona somente a análise da frequência.

A DWT tem sua primeira menção com as Famílias Wavelet de Haar, proposta por Alfred Haar (1909) que é a mais simples das DWT's [20]. Outra importante contribuição ocorreu com os trabalhos de processamento de imagens de Stephane Mallat (1989) [21], que serviram de inspiração para Meyer construir a primeira Wavelet não-trivial [22]. Também baseado nos estudos de Mallat, Ingrid Daubechies construiu um conjunto de bases ortogonais de wavelets não triviais[23], que servem de base para várias aplicações atuais de Wavelets.

\subsection{Transformada Discreta de Fourier}

A Equação (1) apresenta a formulação da DFT (1), em que $\omega$ é a frequência angular do componente, $X(\omega)$ é a representação em frequência do sinal $x[n]$, sendo também conhecido como espectro de $x[n]$.

$$
X(\omega)=\sum_{n=-\infty}^{\infty} x[n] e^{-j \omega n}
$$

Revista Brasileira de Computação Aplicada (ISSN 2176-6649), Passo Fundo, v. 7, n. 3, p. 97-107, out. 2015100 
Segundo Fourier, qualquer função $f(x)$ pode ser aproximadamente descrita na forma da soma de uma série de funções seno e cosseno (2).

$$
f(x)=\begin{aligned}
& a_{n}+a_{1} \operatorname{sen}(x)+a_{2} \operatorname{sen}(2 x)+a_{3} \operatorname{sen}(3 x) \ldots \\
& +b_{1} \cos (x)+b_{2} \cos (2 x)+b_{3} \cos (3 x) \ldots
\end{aligned}
$$

Outra forma de se obter o espectro de frequências de um sinal é aplicando-se a FFT.

\subsection{Transformada Rápida de Fourier}

Em 1969, Cooley e Tukey apresentaram um algoritmo eficiente para o cálculo da DFT [24]. A Equação da FFT divide o somatório em duas partes relacionadas aos índices pares e ímpares chamados de parte real e imaginária respectivamente. O algoritmo matemático da FFT é a melhor forma de calcular a DFT e a sua inversa [25] com menor esforço computacional que a DFT.

De acordo com [26], a DFT de um sinal de tamanho $n$ pode ser reescrita como a soma de duas TF's, cada uma com tamanho igual a $n / 2$, uma originada dos elementos pares do sinal, outra dos elementos impares, considerando as seguintes formulações (3a) a (3d) [27].

$$
\begin{aligned}
& F_{k}=\sum_{j=0}^{n-1} e^{2 \pi i j k / n} f_{i} \\
& F_{k}=\sum_{j=0}^{n / 2-1} e^{2 \pi i k(2 j) / n_{f_{2 j}}}+\sum_{j=0}^{n / 2-1} e^{2 \pi i k(2 j+1) / n_{f_{2 j+1}}} \\
& F_{k}=\sum_{j=0}^{n / 2-1} e^{2 \pi i k\left(\frac{n}{2}\right) f_{2 j}}+W_{k} \sum_{j=0}^{n / 2-1} e^{2 \pi i k\left(\frac{n}{2}\right) f_{2 j+1}} \\
& F_{k}=F_{k}^{e}+W^{k} F_{k}^{o}
\end{aligned}
$$

Nessas formulações $\mathrm{W}=\mathrm{e}^{2 \pi \mathrm{i} / n}$ representa uma constante. $\mathrm{F}_{\mathrm{k}}^{\mathrm{e}}$ é o k-ésimo componente da TF de tamanho $n / 2$ formado pelos componentes pares ("e") da $\mathrm{f}_{\mathrm{j}}$ original. Já $\mathrm{F}_{\mathrm{k}}^{\mathrm{o}}$, representa o k-ésimo componente da $\mathrm{TF}$ de tamanho $n / 2$ formado pelos componentes ímpares ("o") da $\mathrm{f}_{\mathrm{j}}$ original. A variação de $k$ é de 0 até $n$, não restringindo-se somente até $n / 2$. Por conseguinte, as transformadas $\mathrm{F}_{\mathrm{k}}^{\mathrm{e}}$ e $\mathrm{F}_{\mathrm{k}}^{\mathrm{o}}$ são periódicas em $k$ com tamanho $n / 2$. Portanto, cada transformada se repete a cada dois ciclos para obter $F_{k}[27]$.

\subsection{Transformada Wavelet}

A DWT é uma ferramenta que permite decompor um sinal em diferentes componentes de frequências, permitindo assim, estudar cada componente separadamente em sua escala correspondente. Com a Transformada Wavelet é possível manipular recursos em diferentes escalas de forma independente, como suprimir ou reforçar alguma característica particular de um sinal.

O princípio básico da Continuous Wavelet Transform - CWT consiste na convolução da função $f(x)$ a ser generalizada com a função Wavelet $\psi(x)$. Matematicamente, a transformada Wavelet de uma função $f(x)$ é dada por:

$$
C(a, b)=\frac{1}{\sqrt{a}} \int_{-\infty}^{\infty} f(x) \psi *\left(\frac{x-b}{a}\right) d x, \quad a \neq 0
$$




$$
\begin{aligned}
(x) & =\Psi(x-b) \text { translação } \\
\Psi_{a}(x) & =\frac{1}{\sqrt{a}} \Psi\left(\frac{x}{a}\right), a \neq 0 \text { escalonamento } \\
\Psi_{a, b}(x) & =\frac{1}{\sqrt{a}} \Psi\left(\frac{x-b}{a}\right), a \neq 0 \text { translação e escalonamento }
\end{aligned}
$$

Em que a é denominado fator de escala e b é denominado fator de translação (5), ambos pertencentes ao conjunto de números Reais. A função $\Psi(x)$ é chamada de Wavelet-mãe. As funções $\Psi((x-b) / a)$ são as Wavelets derivadas da Wavelet-mãe por translação (6) e mudança de escala ou escalamento (7). Entende-se por escalamento a operação matemática que produz a dilatação ou a compressão de uma função, isso é, mudança de escala da função. O valor de $C(a, b)$ da (1) representa a similaridade entre a função Wavelet e a função analisada $f(x)$. Quanto maior o valor do coeficiente $C(a, b)$, maior a similaridade da função analisada com a função Wavelet. A (8) representa a expressão das operações de translação (6) e escalamento (7) conjuntamente. [28]. No entanto, uma vez que a CWT é obtida a partir de operações de translação e dilatação contínuas sobre a função Wavelet-mãe, uma grande quantidade de informações redundantes são geradas. Assim, uma forma alternativa é a translação e dilatação da Wavelet-mãe a partir de escalas e posições diáticas, ou seja, baseadas em valores de potência de 2, sendo este processo conhecido como Discrete Wavelet Transform - DWT definido como:

$$
D(m, k)=\frac{1}{\sqrt{a_{0}^{m}}} \sum_{n} x(n) g\left(\frac{k-n b_{0} a_{0}^{m}}{a_{0}^{m}}\right)
$$

Em que os parâmetros $a$ e $b$ da equação (5) são funções de $m$, sendo $a=e b=e k$, uma variável inteira relacionada ao tamanho de uma dada amostra do sinal de entrada [29];

\subsection{Famílias de Transformadas Wavelet}

A DWT possui uma ampla variedade de filtros específicos, sendo que cada filtro apresenta

\begin{tabular}{|c|c|c|c|}
\hline Família & Suporte & Fase & Observação \\
\hline Haar & 2 & Linear & é a mais simples das Wavelets criada por Alfred Haar \\
\hline Daubechies & par, maior que 4 & não linear & $\begin{array}{l}\text { resposta ao impulso maximally flat, criada por Ingrid } \\
\text { Daubechies }\end{array}$ \\
\hline Symmlets & par, múltiplo de 8 & não linear & resposta ao impulso mais simétrica \\
\hline Coiflets & Par múltiplo de 6 & quase linear & $\begin{array}{l}\text { resposta ao impulso quase simétrica, criada por } \\
\text { Ronald Coifman }\end{array}$ \\
\hline Vaidyanathan & 24 & não linear & otimizada para voz, criada por P. P. Vaidyanathan \\
\hline Beylkin & 18 & não linear & otimizada para áudio em geral \\
\hline
\end{tabular}
características próprias, assim como diferentes suportes para cada filtro. A Tabela 2 resume de maneira geral as particularidades de cada uma das principais famílias de filtros Wavelets.

Tabela 2: Famílias Wavelets

Fonte: [30]. 


\section{Desenvolvimento}

O presente artigo apresenta um estudo comparativo entre as complexidades dos algoritmos da DFT e DWT. Tal comparação se deu contrapondo-se os valores de complexidades computacionais inerentes a cada transformada, além de análises que envolveram as vantagens e desvantagens de cada transformada em relação à outra.

Dessa forma, para a comparação dos algoritmos das transformadas propostas neste trabalho, foi adotada a seguinte metodologia:

a) As complexidades computacionais assintóticas em termos de notação $\mathrm{O}$, foram consideradas a partir de algoritmos clássicos dessas ferramentas, amplamente discutidos e utilizados em trabalhos acadêmicos de variadas áreas de conhecimento.

b) Foi analisada, para a DWT, o filtro de Haar e suas principais características;

c) Comparação entre resolução em frequência alcançada pela DFT e a resolução tempo/frequência da DWT.

Por fim, foram utilizadas duas bibliotecas em linguagem Java, chamadas de JTransform e JWave, as quais implementam diversas formas de transformadas, tendo como elementos principais a DFT, FFT e DWT, com a possibilidade de utilização das famílias Wavelets de Haar, Daubechies e Coiflets, sendo que neste trabalho foi utilizada somente a família de Haar. Foram feitos experimentos com as diversas transformadas, aplicando-as a arquivos de áudio no formato .wav com diferentes tamanhos, de forma a evidenciar as diferenças em termos de complexidade entre os algoritmos e também a diferença em tempo de execução para diferentes tamanhos de entrada.

\section{Resultados e Discussão}

É possível perceber que, quando comparados os esforços computacionais dos três algoritmos DFT, FFT e DWT, verifica-se que o primeiro e mais antigo dos três é o que tem maior complexidade $O\left(n^{2}\right)$. O segundo que é uma forma mais eficiente de se calcular a Transformada de Fourier, a complexidade algorítmica diminui para $O(n \log n)$. Por fim, a DWT tem uma complexidade ainda menor que FFT, pois pode ser calculada usando apenas $O(n)$ multiplicações.

No entanto o tempo de execução de um algoritmo é diretamente afetado pelas entradas de dados, ou seja, o sinal que ele irá processar, conforme pode ser visualizado na Figura 2:

Figura 2: Gráfico do número de operações realizadas em relação ao tamanho da entrada n do sinal

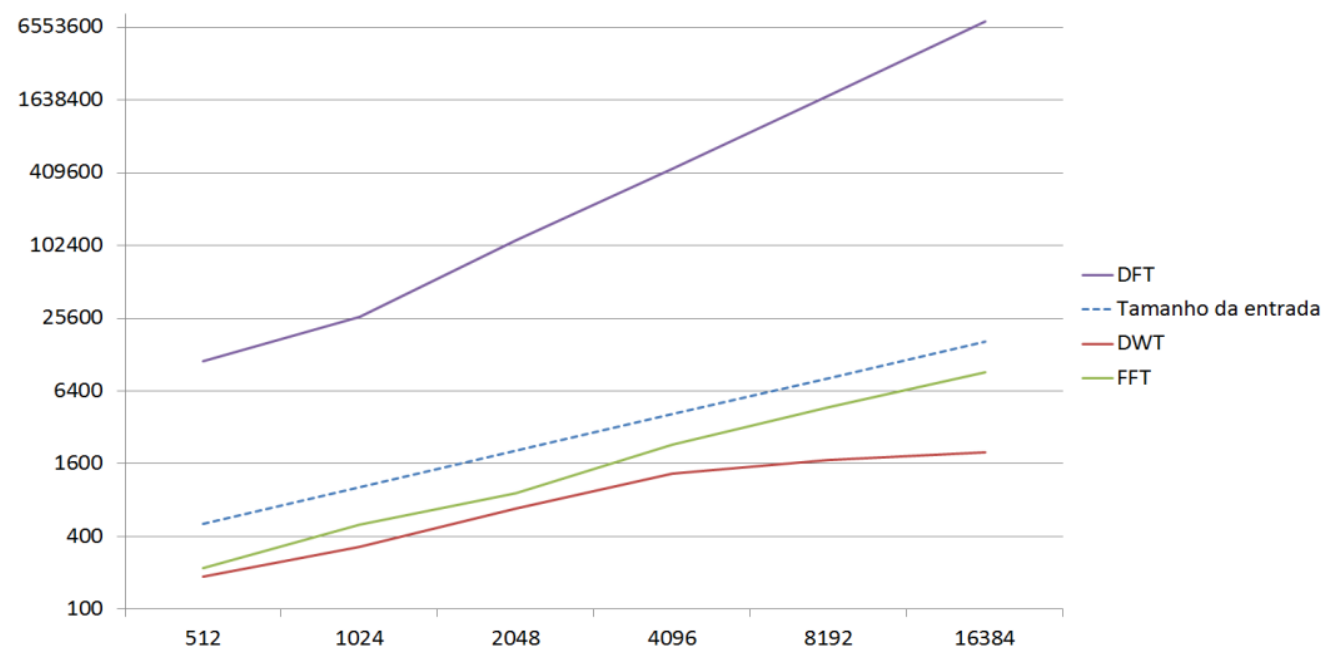

Revista Brasileira de Computação Aplicada (ISSN 2176-6649), Passo Fundo, v. 7, n. 3, p. 97-107, out. 2015103 
Outro ponto a ser considerado é a evolução tecnológica vivenciada nos dias atuais. O aprimoramento e surgimento de novos hardwares, microprocessadores com maior poder de processamento, memórias mais rápidas e com maior capacidade de armazenamento, podem amenizar o tempo de execução do algoritmo. No entanto, apesar de altamente dependente do hardware utilizado, o tempo de execução pode ser uma métrica interessante para avaliação de desempenho do algoritmo, principalmente com objetivos didáticos e comparativos. Dessa forma, foram executados os algoritmos das transformadas em estudo para sinais reais de áudio de diferentes tamanhos, e cronometrados seus tempos de execução, de forma que os valores temporais de cada série de execução são mostrados na Tabela 3. O computador utilizado para a execução dos algoritmos foi um notebook da marca Dell, com processador Intel Core i7, 8 GB de memória RAM e placa de vídeo dedicada NVIDIA de 2 GB. Os algoritmos utilizados foram os implementados nas bibliotecas JWave e JTransform, ambos desenvolvidos na linguagem Java.

\begin{tabular}{ccc} 
Tabela 3: Tamanho do sinal e tempo de execução para as transformadas DWT, FFT \\
\hline Transformada & Tamanho do sinal de entrada $(n)$ & Tempo $(\mu s)$ \\
\hline DWT & 512 & 186 \\
& 1024 & 330 \\
& 2048 & 678 \\
& 4096 & 1322 \\
& 8192 & 1716 \\
& 16384 & 1985 \\
\hline FFT & 512 & 220 \\
& 1024 & 498 \\
& 2048 & 913 \\
& 4096 & 2296 \\
& 8192 & 4677 \\
& 16384 & 9148 \\
\hline
\end{tabular}

Fonte: Os autores.

Além das complexidades computacionais inerentes aos algoritmos, foram ainda adotadas como critérios de comparação, características específicas a cada transformada. A transformada Wavelet, conforme já citado anteriormente tem uma variada gama de filtros particularmente desenvolvidos para problemas específicos, como o caso do filtro de Vaidyanathan para voz, o filtro de Beylkin otimizado para faixas de áudio em geral e o filtro Spikelet especializada para o reconhecimento de padrões de picos. De outra forma, as transformadas de Fourier são mais genéricas uma vez que não têm tal diferenciação e especificidade para cada problema. Dessa forma, em um primeiro momento pode parecer que as Wavelets possuem grandes vantagens em relação às transformadas e Fourier, no entanto caso não sejam conhecidas às características do problema em estudo, uma escolha arbitrária da família de filtros para a transformada Discreta Wavelet pode acarretar em grandes perdas de partes significativas de um sinal, fazendo com que, apesar de mais custosa em termos computacionais, as transformadas Discreta de Fourier possam se mostrar mais eficazes para tais situações.

Outra característica proposta como forma de comparação são as resoluções em frequência da transformada Discreta de Fourier e a resolução tempo/frequência da transformada Discreta Wavelet. A resolução 
tempo/frequência proporcionada pela transformada Discreta Wavelet possibilita uma série de análises diferenciadas em relação a Fourier, uma vez que insere a possibilidade de localização temporal juntamente com a localização em frequência. Por exemplo, se duas notas musicais forem tocadas sequencialmente em um instrumento musical, a partir da transformada Discreta de Fourier seria possível determinar quais foram essas notas, sem, entretanto determinar a ordem ou a sequência em que essas foram tocadas. A resolução tempo/frequência das transformadas Discretas Wavelets por sua vez, possibilita tal identificação.

Dentre os trabalhos relacionados encontrados na literatura o estudo comparativo de desempenho das transformadas discretas de Fourier e Wavalets aplicados para detecção, classificação e localização de falhas em linhas de transmissão [31], reforça os resultados obtidos neste estudo, que indica que as características de cada transformada podem ser adequadas às resoluções de problemas distintos, como exemplo do trabalho citado que mostra que a transformada Discreta Wavelet obteve melhor resultado na classificação e nas estimativas de distâncias de falhas. A transformada Discreta de Fourier por sua vez obteve melhor desempenho na previsão de um tipo específico de falha. Estes fatos levaram os autores a sugerir uma abordagem híbrida que utiliza a combinação de ambas as transformadas. Outras análises das transformadas são encontradas na literatura como a que apresenta um estudo comparativo da transformada Wavelet no reconhecimento de padrões da íris humana [32]. Outro exemplo é o estudo comparativo sobre filtragem de sinais instrumentais usando transformadas de Fourier e Wavelet [33], todos enfatizam além das diferenças as potencialidades de cada transformada.

\section{Conclusão}

De uma forma geral, a teoria da complexidade apresentada neste trabalho demostra como a eficiência dos algoritmos é dependente das entradas e das equações matemáticas que eles realizam no momento em que são executados, e que existem problemas que são impraticáveis com a atual tecnologia em função tempo necessário para sua completude.

O PDS é um recurso importante e poderoso na resolução de vários problemas em diversas áreas da ciência. É possível também verificar que as soluções propostas por meio do processamento de sinais são fortemente embasadas na fundamentação matemática e que esta formalização também permite avaliar o esforço computacional de cada algoritmo.

Assim, tendo em vista as características específicas das abordagens, é importante levar em consideração o tipo de problema a ser resolvido para se adotar a melhor solução possível. Especificamente com relação às TD’s analisadas, entende-se que a DWT é a mais rápida em comparação com a FFT, que por sua vez é mais rápida que DFT, portanto os algoritmos implementados utilizando Wavelets exigem menor esforço computacional que os demais apresentados. Entretanto, a respeito da complexidade de cada transformada, é importante ressaltar o fato que a escolha da transformada deve levar em consideração outros aspectos como característicos do problema e o tipo de resolução pretendido, somente em frequência ou tempo/frequência.

Durante as pesquisas, verificou-se a existência de duas bibliotecas para a Linguagem Java que implementam as Transformadas Discretas de Fourier e Wavelet, proporcionando assim um código mais limpo, e um desenvolvimento rápido de aplicações, tendo em vista que os cálculos são implementados por meio dos métodos das bibliotecas chamadas JWave e JTransform que por sua vez recebem as entradas por parâmetros, assim uma opção para iniciar a utilizar as técnicas de PDS

Por fim, espera-se que este trabalho sirva como uma base inicial para interessados em conhecer técnicas de PDS e que também possa contribuir como referencia para trabalhos futuros por meio das informações nele reunidas.

\section{Agradecimentos}

Agradecimentos a Capes pelo apoio financeiro por intermédio das bolsas de mestrado e a UEL por fornecer os recursos necessários para o desenvolvimento do trabalho. 


\section{Referências}

[1] KEMPER, G.; IANO, Y. An Audio Compression Method Based on Wavelets Subband Coding. in Latin America Transactions, IEEE (Revista IEEE America Latina), vol. 9, n. 5, p. 610-621, Sept. 2011.

[2] PAIVA, J. P. L. M. et al. Adaptive wavelet EMG compression based on local optimization of filter banks. J. Physiological Measurement, Inst. of Physics and Eng. in Medicine. vol. 29, n.7, p. 843-856, July 2008.

[3] REIS, R. S. L.; MACHADO, P. C. M.; LEMOS, R. P. A transformada wavelet no tratamento de sinais sonoros de aves noturnas. In: CONGRESSO DE MATEMÁTICA APLICADA E COMPUTACIONAL, CMAC-Norte 2012. Anais... Macapá-AP: Unifap, 2012. p. 31-34. Disponível em:

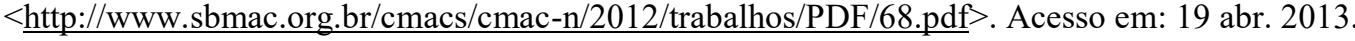

[4] GEROLAMO, R. O. P.; KAYANO, M. T. Variações do ciclo anual da temperatura da superfície do mar no pacífico tropical. Rev. Brasileira de Meteorologia, São José dos Campos-SP, v. 25, n. 2, p. 237-247, jun/2010.

[5] CHEN, B.; ZHAO, J. An adaptive and secure audio watermarking algorithm robust to MP3 compression. In: Instrumentation and Measurement Technology Conference, I2MTC '09. IEEE, Proceedings... Singapore, 2009. p. 629-632.

[6] MARCELlin, M. W. et al. An Overview of JPEG2000. In: Data Compression Conference, Proceedings... Snowbird, Utah, Mar. 2000. p. 523-541.

[7] MEDEIROS, F. A.; SUSANNA, R. Jr. Análise de Fourier para detecção de defeitos localizados na camada de fibras nervosas da retina com a polarimetria a laser. Arquivos Brasileiros de Oftalmologia, São Paulo, v. 66, n. 6, dez. 2003. p. 846-853.

[8] SHI, B. et al. $360^{\circ}$ Fourier Transform Profilometry in Surface Reconstruction for Fluorescence Molecular Tomography. IEEE Journal of Biomedical and Health Informatics, Bethesda, Maryland, v. 17, n. 3, may 2013, p. 681-689.

[9] KAZMI, S. B.; AIN, Q.; JAFFAR, M. A. Wavelets Based Facial Expression Recognition Using a Bank of Neural Networks. In: INTERNATIONAL CONFERENCE ON FUTURE INFORMATION TECHNOLOGY (FUTURE TECH), 5. Proceedings... Busan, 2010. p. 1-6.

[10] AGGOUN A. Compression of 3D Integral Images Using 3D Wavelet Transform. IEEE/OSA Journal of Display Technology, Piscataway, New Jersey, v.7, n.11, p.586-592, Nov. 2011.

[11] SILVA, Marcelo M.; MELlo, Luiz A. R. da S. Avaliação de algoritmos de detecção de espaços espectrais brancos para aplicações de rádio cognitivo. 2014. 129f. Dissertação Mestrado em Engenharia Elétrica - Departamento de Engenharia Elétrica, PUCRJ, Rio de Janeiro, 2014

[12] RODRIGUES, Jorge; CORTE-REAL, Luís; CARVALHO, Pedro. Análise da variação dinâmica da complexidade de um algoritmo de tracking. 2012. 94f. Dissertação (Mestrado Integrado em Engenharia Eletrotécnica e Computadores) - Feup, Porto, 2012.

[13] MANBER, Ubi. Introduction to algorithms: A creative approach. Boston, MA, USA, Addison-Wesley Longman Publishing Co., Inc., 1989.

[14] GOODRICH, Michael T.; TAMASSIA, Roberto. Estruturas de Dados e Algoritmos em Java. 4. ed. Porto Alegre: Bookman, 2007.

[15] HeIneman, G. T.; POLliCE, G.; SElKOW, S. Algoritmos: O Guia Essencial. 2. ed. Rio de Janeiro: Alta Books, 2009.

[16] CORMEN, T. H. et al. Algoritmos: Teoria e Prática. 2. ed. Rio de Janeiro: Campus, 2002.

[17] SAHNI, S. Data Structures, Algorithms and Applications in C++. 2. ed. Hyderabad, IN: Univ. Press, 2005.

[18] HAYKIN, S. S.; VAN VEEN, B. Sinais e Sistemas. Porto Alegre: Bookman, 2001.

[19] LYON, Douglas. The Discrete Fourier Transform, Part 1. Journal of Object Technology. ETH Zurich, Chair of Software Engineering, v. 8, n. 3, p. 17-26, May-June 2009.

Revista Brasileira de Computação Aplicada (ISSN 2176-6649), Passo Fundo, v. 7, n. 3, p. 97-107, out. 2015106 
[20] HAAR, A. ZurTheorie der Orthogonalen Funktionen Systeme. Mathematische Annalen v. 69, n. 3 pp. 331371. 1910.

[21] MALLAT, S. G. A Theory for Multiresolution Signal Decomposition: The Wavelet Representation. IEEE Transctions on Pattern Analysis and Machine Intelligence, Illinois, v. 11, n. 7, p. 674-693, Jul/1989.

[22] MEYER, Y. Wavelets: Algorithms and Applications. Philadelphia: Soc. for Ind. and Aplied Math., 1993.

[23] DOUBECHIES, I. The Wavelet Transforma, Time-Frequency Localization and Signal Analysis. IEEE Transactions on Information Theory, Piscataway: NJ, v. 36, n. 5, p. 961-1005, Sep/1990.

[24] COOLEY, J. W.; LEWIS, P. A. W.; WELCH, P. D. The Fast Fourier Transform and its Application. IEEE Transactions on Audio and Eletroacoustics, v. 12, n.1, March/1969, p. 27-34.

[25] COOLEY, J. W.; TUKEY, J. W. An Algorithm for the Machine Calculation of Complex Fourier Series. Jornal Mathematics of Computation, Pub:American Mathematical Society, Providence: RI, v. 19, n. 90, p. 297-301, Apr/1965.

[26] DANIELSON, G. C.; LANCZOSC. Some improvements in practical Fourier analysis and their application to X-ray scattering from liquids. J. of Franklin Institute, v. 233, n.5, may/1942, p.435-452 and 365-380.

[27] PORFIRIO, Ronie P. et al. Processamento de imagens multitemporais usando a transformada rápida de Fourier (FFT) na dimensão do Tempo. In: SIMPÓSIO BRASILEIRO DE SENSORIAMENTO REMOTO, 14. Anais... Natal: Inpe, 2009. p. 7071-7078.

[28] MALLAT, Stéphane G. A Wavelet tour of signal processing. San Diego, California: Academic Press, 1998.

[29] REIS, Agnaldo J. R.; SILVA, Alexandre P. A. Aplicação da transformada Wavelet discreta na previsão de carga a curto prazo via redes neurais. Rev. Controle \& Automação, Campinas, v.15, n.1, jan.-mar. 2004.

[30] SOUZA, Leonardo M. Detecção inteligente de patologias na laringe baseada em máquinas de vetores de suporte e na transformada Wavelet. Dissertação Mestrado Física Aplicada, Instituto de Física de São Carlos, São Carlos, 2010.

[31] DAS, Debrup; SINGH, Neeraj Kumar; SINHA, Avinash K. A comparison of Fourier transform and wavelet transform methods for detection and classification of faults on transmission lines. In: IEEE Power India Conference, Proceeding... IEEE 2006. p. 7.

[32] CASTELANO, Célio R.; GONZAGA, Adilson. Estudo comparativo da Transformada Wavelet no reconhecimento de padrões da iris humana. Dissertação (Mestrado em Engenharia Elétrica) - EESC/USP, São Paulo, 2006.

[33] GALVÃO, Roberto K. H. et al. Estudo Comparativo sobre filtragem de sinais instrumentais usando transformadas de Fourier e Wavelet. Química Nova, São Paulo, v. 24, n. 6, p. 874-884, 2001. 